\title{
APIANO DE ALEJANDRÍA, TRADUCTOR (BC IV 45 Y V 191) *
}

JOSÉ B. TORRES

Universidad de Navarra

jtorres@unav.es

Este trabajo defiende que Apiano considera como traducción dos pasajes de su obra (cf. BC IV 45 y $\mathrm{V}$ 191). Las dos traducciones poseen caracteres distintos que han de ser explicados a la luz de las teorías antiguas sobre la traducción.

Palabras clave: Apiano; traducciones griegas del latín.
This paper defends that Appian regards as translations two passages in his work (cfr. BC IV 45 and V 191). Both translations show different features which may be explained from the point of view of ancient theories concerning translation.

Keywords: Appian; Greek translations from Latin.

1. En el siglo segundo de la era cristiana, Apiano de Alejandría hizo de Roma el tema de su obra. Su Historia romana comparte esta materia con los escritos de toda una serie de historiadores griegos de su época: Dión Casio, Herodiano o Zósimo son aquellos de los que hemos conservado los textos principales ${ }^{1}$. La existencia de esta corriente dentro de la historiografía helénica sirve como primer recordatorio de que, en época imperial, la cultura y literatura griegas se hallaban mucho más imbricadas con Roma de lo que a veces puede pensarse.

En este trabajo no voy a tratar sobre las fuentes latinas de Apiano, cuestión que ya ha sido discutida en numerosas ocasiones por la bibliografía ${ }^{2}$.

* Este trabajo forma parte del proyecto "Graecia capta. El influjo de la literatura latina en la cultura y literatura de Grecia (I)", financiado por la Universidad de Navarra (PIUNA 11328301) y el Ministerio de Educación y Ciencia (HUM 2004 - 01478 / FILO). Deseo agradecer a los doctores Sánchez-Ostiz y Lluch Villalba la atención con que leyeron versiones previas de esta colaboración.

1 Me refiero únicamente a autores de época imperial, motivo por el que dejo de lado los nombres de Polibio o Dionisio de Halicarnaso. De otro lado, a esta lista también podría haberse añadido a Plutarco por sus biografías de figuras romanas.

2 Para una panorámica general del asunto, cf. la exposición de A. Sancho Royo, Apiano. Historia romana. I, Madrid, 1980, pp. 13-16. La cuestión de las fuentes de Apiano recibe un tratamiento pormenorizado en M. Hose, Erneuerung der Vergangenheit. Die Historiker im 
En su lugar voy a referirme a Apiano como latinista ${ }^{3}$ y, más en concreto, como traductor del latín al griego.

Cuando oímos hablar sobre traducciones en la Antigüedad, pensamos espontáneamente en las múltiples traducciones y adaptaciones romanas de originales griegos ${ }^{4}$. No en vano la historia de la literatura latina escrita se inicia precisamente con la versión de la Odisea compuesta por Livio Andronico. Sin embargo, el canal que conecta las literaturas de Grecia y Roma funcionó en los dos sentidos. Igual que hubo traducciones latinas de autores griegos, existieron también versiones griegas de autores romanos. La más famosa entre las conservadas ${ }^{5}$ es la traducción de la Égloga IV de Virgilio incluida en la Oratio ad sanctorum coetum que compuso el emperador Constantino, según el testimonio de Eusebio de Cesarea (Vita Constantini IV 32) ${ }^{6}$. Pero ésta no es la única traducción griega de un original latino, según destaca la bibliografía que existe sobre el tema, ciertamente no muy abundante ${ }^{7}$.

2. Apiano no es sólo uno de los autores griegos que toman Roma como tema o que escriben bajo el influjo de la literatura latina, en su caso de los

Imperium Romanum von Florus bis Cassius Dio, Stuttgart-Leipzig, 1994 (cf. pp. 142-355: "5. Appian").

3 Cf. J. Hering, Lateinisches bei Appian, Weida, 1935; E. Famerie, Le latin et le grec d'Appien: contribution à l'étude du lexique d'un historien grec de Rome, Ginebra, 1998.

4 Sobre el tema, cf. A. Seele, Römische Übersetzer: Nöte, Freiheiten, Absichten. Verfahren des literarischen Übersetzens in der griechisch-römischen Antike, Darmstadt, 1995.

5 Sabemos que Polibio, liberto de Claudio, compuso una traducción o paráfrasis de la Eneida (cf. Séneca, Cons. ad Pol. VIII 2). De un pasaje de Macrobio (Sat. V 2, 4) parece deducirse que Pisandro de Laranda, en el siglo III, tradujo al griego la Eneida (cf. M. Hose, "Die römische Liebeselegie und die griechische Literatur", Philologus 138, 1994, pp. 67-82;

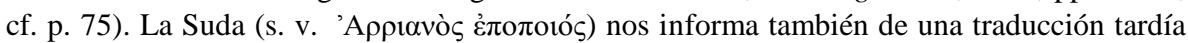
de las Geórgicas. No se conserva ninguna de estas versiones.

${ }^{6}$ Sobre esta traducción existe abundante bibliografía. Cf. p. ej. C. Monteleone, L'Egloga Quarta da Virgilio a Costantino: critica del testo e ideologia, Manduria, 1975; E. Fisher, "Greek Translations of Latin Literature in the $4^{\text {th }}$ cent. A. D.", YCS 27 (1982), pp. 173-215 (cf. pp. 177-182); B. Rochette, "Bilinguisme, traductions et histoire des textes dans l'Orient grec (I-IV siècle après J.C.)", RHT 27 (1997), pp. 1-28 (cf. pp. 20-26).

7 Una referencia clásica es V. Reichmann, Römische Literatur in griechischer Übersetzung, Leipzig, 1943. Entre los trabajos posteriores pueden destacarse los ya citados de Fisher y Rochette.

EMERITA (EM) LXXIV 1, enero-junio 2006 pp. 17-28 ISSN 0013-6662 
historiadores romanos ${ }^{8}$. La cuestión que ahora nos interesa es que, en dos lugares de su obra, Apiano declara o parece declarar que el texto que está incluyendo es traducción de sus fuentes latinas. Ambos lugares se encuentran en los libros dedicados a las guerras civiles de Roma (Bella Ciuilia $=$ $B C)$, la porción de su Historia romana que mejor se conserva ${ }^{9}$.

El primer caso lo encontramos en el libro cuarto de $B C$, donde se relata el enfrentamiento de Marco Antonio y Octavio con los asesinos de César. Entre $B C$ IV 31 y 44 se incluye el texto de la proscripción decretada en el año 43 a. C. por Lépido, Antonio y Octavio contra los ciudadanos considerados traidores a Roma ${ }^{10}$. Al final de la misma, el autor declara:

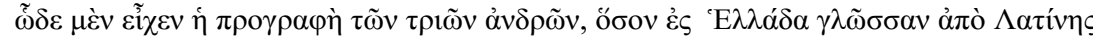
$\mu \varepsilon \tau \alpha \beta \alpha \lambda \varepsilon \tilde{i} v$, "El escrito de proscripción de los triunviros era de este tenor, en la medida en que se puede verter a la lengua griega desde la latina" ( $B C$ IV 45).

Un caso que podemos percibir como análogo (al menos en una primera lectura) aparece en el libro siguiente de la obra, cuyo tema general es el conflicto entre Antonio y Octavio. En BC V 171 Apiano comienza a narrar un encuentro mantenido entre Lucio Antonio (hermano del triunviro) y Octavio Augusto en el año 40 a. C. Entre $B C$ V 176 y 190 expone los discursos pronunciados por ambos, según hace en diversos lugares de su obra ${ }^{11}$. El pasaje se diferencia de otros en los que Apiano incluye intervenciones en estilo directo por el añadido final:

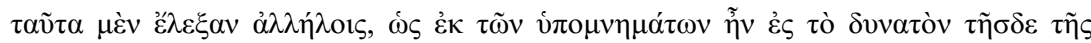

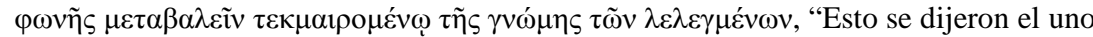
al otro, según se podía verterlo a partir de los comentarios, de acuerdo con las posibilidades de esta lengua, deduciéndolo yo de la idea general de lo dicho" (BC V 191).

8 El conocimiento de la literatura latina que tenía Apiano debía de ser más amplio. Por ejemplo, parece probable que en $B C$ IV 41 presuponga el conocimiento de la Eneida (II 721 ss.). Cf. la evidencia reunida por M. Hose (Erneuerung..., p. 333) sobre citas y alusiones literarias en Apiano.

9 Las Guerras Civiles ocupan cinco libros del total. Han llegado hasta nosotros, además, íntegros o en epítomes, otros diecinueve libros. Como edición de referencia, cf. la publicada por la editorial Teubner (P. Viereck, A.G. Roos y E. Gabba [eds.], Appiani Historia Romana. I, Leipzig, 1962; L. Mendelssohn y P. Viereck [eds.], Appiani Historia Romana. II, Leipzig, 1902).

10 Hoy por hoy parece haber acuerdo en considerar el decreto de proscripción como auténtico. Cf. la evidencia reunida por Famerie, Le latin et le grec..., p. 30, n. 114; p. 90, n. 112.

11 Sobre los discursos en la Historia romana de Apiano, cf. lo que indica Hose, Erneuerung..., pp. 268-273.

EMERITA (EM) LXXIV 1, enero-junio 2006 pp. 17-28 ISSN 0013-6662 
3. Martin Hose ${ }^{12}$ comenta que este segundo caso es diferente del primero: en

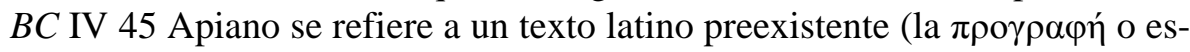
crito de proscripción) que él vierte al griego; en cambio, en $B C$ V 191 no se alude a un texto latino escrito que contuviera los discursos sino que se expone que el autor hubo de deducir el contenido de las intervenciones de Lucio Antonio y Octavio a partir de los comentarios ( interpretación de $B C$ V 191 es importante dentro de la argumentación de Hose porque le permite ilustrar que Apiano no se limita a reproducir o traducir los discursos que encuentra en sus fuentes sino que los reelabora de acuerdo con sus intereses.

Pienso que, en efecto, entre los dos pasajes existe esa diferencia a la que se refiere Hose: en el primer caso hay versión de un texto escrito y en el segundo no. Nuestro concepto actual de "traducción" sólo es aplicable al primero de estos pasajes ${ }^{14}$ pero no al segundo. Ahora bien, pienso también ( $p a-$ ce Hose) que, para el autor, las operaciones realizadas por él en un caso y otro eran igualmente traducciones. Intentaré profundizar en la cuestión a partir de alguna observación lingüística.

4. Apiano emplea en los dos pasajes ( $B C$ IV 45 y V 191) el mismo verbo para referirse a su actividad, y éste es un punto al que otros trabajos quizá no han dedicado atención suficiente ${ }^{15}$. El verbo en cuestión es $\mu \varepsilon \tau \alpha \beta \alpha \lambda \varepsilon i v$, infinitivo de aoristo de $\mu \varepsilon \tau \alpha \beta \alpha ́ \lambda \lambda \omega$, verbo que, según indica el Lexicon de

12 M. Hose, Erneuerung..., p. 173, n. 19; p. 269, n. 10. Cf., en sentido contrario, E. Gabba, Appiani Bellorum Ciuilium Liber Quintus, Florencia, 1970, p. 77; idem, «Appiano traduttore in B.C., V 191», en VVAA., Studi di storiografia antica: in memoria di Leonardo Ferrero, Turín, 1971, pp. 185-189.

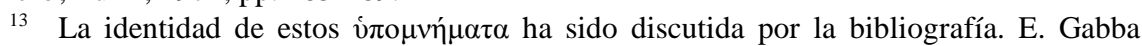
(Appiani..., pp. XVII-XXIII; «Appiano traduttore...», p. 188) opina que la fuente última de Apiano eran las acta urbis o populi diurna acta. Resulta muy sugerente la hipótesis de A.M. Gowing («Appian and Cassius' Speech before Philippi», Phoenix 44, 1990, pp. 158-181, cf. p. 180, n. 63), según el cual los comentarios a que alude Apiano habrían sido las Memorias de Augusto (cf. infra).

${ }_{14}$ Por cierto que el hecho de que Apiano traduzca el texto de la proscripción es uno más de los argumentos que permiten afirmar que este autor poseía competencia lingüística en latín. Cf. la bibliografía citada en n. 3 .

15 Cf. Hose, Erneuerung..., p. 269, n. 10. Tampoco Gabba (Appiani..., «Appiano traduttore ...») comenta la coincidencia en el empleo de $\mu \varepsilon \tau \alpha \beta \alpha \lambda \varepsilon \tilde{v}$.

EMERITA (EM) LXXIV 1, enero-junio 2006 pp. 17-28 ISSN 0013-6662 
Liddell-Scott, puede tener ocasionalmente el valor de 'traducir' ${ }^{16}$. Es oportuno indicar que, según se deduce del Liddell-Scott, el verbo sólo adopta este significado de manera esporádica; el único ejemplo del mismo que propone esta obra procede de las Antigüedades Judías de Flavio Josefo

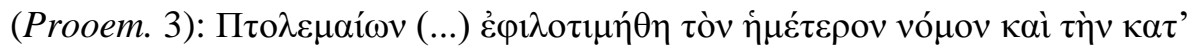

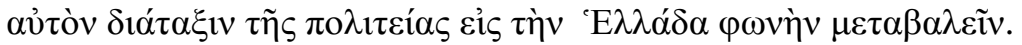

Sucede además que $\mu \varepsilon \tau \alpha \beta \alpha \dot{\lambda} \lambda \lambda \omega$, empleado por Apiano en estos dos pasajes de $B C^{17}$, no es ninguno de los verbos que se utilizaban de manera habitual en griego para expresar el concepto 'traducir'. Si acudimos a un diccionario inverso ${ }^{18}$, comprobaremos que este significado se expresaba por medio de otros significantes:

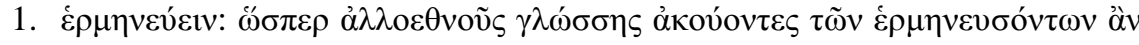
$\delta \varepsilon \eta \theta \varepsilon \tilde{\varepsilon} v$ (D.H., Th. 49);

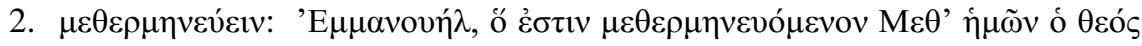
(Ev. Matt. I 23);

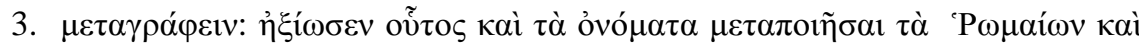

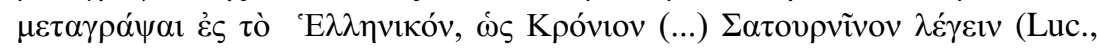
Hist. Conscrib. 21);

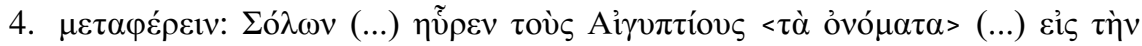

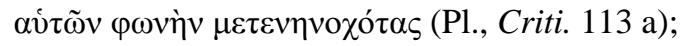

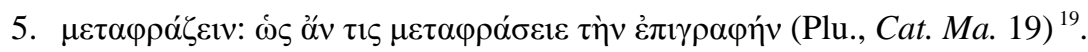

De estos cinco verbos, sólo $\mu \varepsilon \tau \alpha \varphi \varepsilon ́ p \omega$ se emplea en la Historia romana de Apiano, aunque nunca con el valor de "traducir". Los otros cuatro verbos no se documentan nunca en el conjunto de la obra ${ }^{20}$.

En resumen, en los dos pasajes analizados ( $B C$ IV 45 y $B C$ V 191) Apia-

16 Cf. H.G. Liddell, R. Scott y H. Stuart Jones (eds.), A Greek-English Lexicon, Oxford, $1940^{9}$, s. v. $\mu \varepsilon \tau \alpha \beta \alpha ́ \lambda \lambda \omega$ (II b).

17 En el conjunto de su obra, Apiano emplea $\mu \varepsilon \tau \alpha \beta \alpha ́ \lambda \lambda \omega$ con el significado de 'traducir' sólo en estos dos pasajes. Cf. E. Famerie (ed.), Concordantia in Appianum, Hildesheim, 1993, s. v.

18 Cf. W. Pape y M. Sengebusch, Handwörterbuch der griechischen Sprache. IV. Deutsch-griechisch Wörterbuch, Braunschweig, $1875^{3}$, s. v. "Übersetzen".

19 He identificado además en Plutarco (Cic. 40) un pasaje en el que alternan $\mu \varepsilon \tau \alpha \varphi \rho \alpha ́ \zeta \omega$

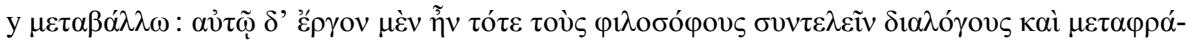

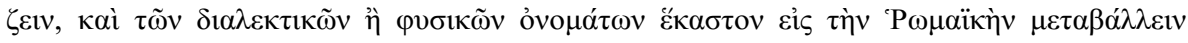
$\delta i \alpha ́ \lambda \varepsilon \kappa \tau o v$

20 Cf. Famerie, Concordantia...., s. vv.

EMERITA (EM) LXXIV 1, enero-junio 2006 pp. 17-28 ISSN 0013-6662 
no designa su actividad como traductor por medio de un verbo ( $\mu \varepsilon \tau \alpha \beta \alpha ́ \lambda \lambda \omega)$ que, aun poseyendo en ocasiones el valor de "traducir", es distinto de los usados habitualmente en griego para la expresión de este significado. De ello puede deducirse que la coincidencia en el empleo de esta voz marcada, no habitual ( $\mu \varepsilon \tau \alpha \beta \alpha ́ \lambda \lambda \omega)$, en los dos lugares de las Guerras civiles (BC IV 45 y $B C$ V 191) apoya la hipótesis y refuerza la idea de que existe una similitud de sentido entre ambos pasajes. Como decía más arriba, entiendo que Apiano parece concebir en los dos casos su actividad como esencialmente idéntica: como traducción.

5. No obstante, para avanzar en la argumentación hemos de reparar en el hecho de que hay diferencias entre los marcos predicativos de $\mu \varepsilon \tau \alpha \beta \alpha \lambda \varepsilon \tilde{v} v$ en uno y otro lugar. Posiblemente, esas diferencias sintácticas guardan relación con formas distintas de ejercer la acción de traducir.

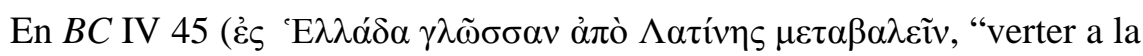
lengua griega desde la latina"), dos complementos circunstanciales dependientes del infinitivo indican las lenguas de llegada y de partida de la

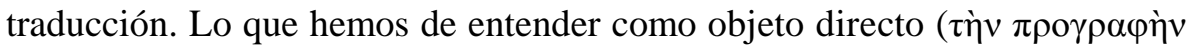
$\tau \tilde{\omega} v \tau \rho \iota \tilde{\omega} v \dot{\alpha} \nu \delta \rho \tilde{\omega} v)$ se reconstruye a partir de la oración principal.

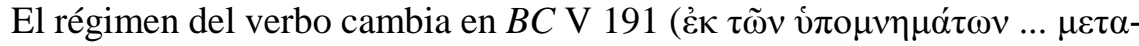
$\beta \alpha \lambda \varepsilon i v$, "verterlo ... a partir de los comentarios"). En este segundo caso, el

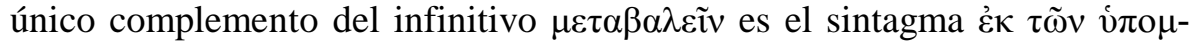

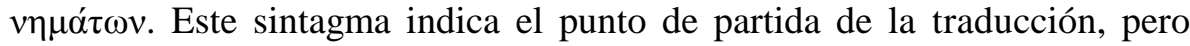
evidentemente este punto de partida no es ahora la lengua desde la que se

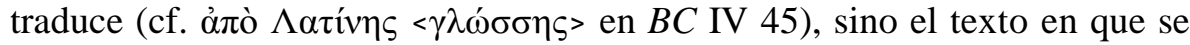
basa Apiano a la hora de hacer su versión de los discursos: los comentarios ( $(\pi \circ \mu v \eta ́ \mu \alpha \tau \alpha)$, que en ningún caso hay que entender, con algunos traductores $^{21}$, como el objeto directo elidido de $\mu \varepsilon \tau \alpha \beta \alpha \lambda \varepsilon i ̃ v$. Una vez más (cf. lo dicho en el párrafo anterior), el objeto directo del verbo se restituye a partir de la oración principal, donde $\tau \alpha \tilde{v} \tau \alpha$ se refiere a los parlamentos pronunciados por Lucio Antonio y Octavio Augusto.

21 Como por ejemplo Sancho Royo (Apiano. Historia romana. III, Madrid, 1985, p. 268), quien propone traducir así $B C$ V 191: «en la medida en que me fue posible conocer el espíritu de lo que se dijo a partir de los comentarios, y verterlos a nuestra lengua» (la cursiva es mía). Me parece más ajustada la traducción de Gabba (Appiani..., p. 272): «questo si dissero fra di loro, per quanto dai commentari, argomentando il significato delle cose dette, è possibile trasferirle in questa lingua». 
6. Lo que parece estar aquí en juego es el concepto antiguo de traducción, concepto que, como hemos de ver seguidamente, aceptaba la existencia de traducciones de tipo distinto; más aún, este concepto operativo en la Antigüedad reconocía como traducciones cosas que para nosotros no entran en esta categoría. Por supuesto, podríamos decir simplemente, aplicando terminología de nuestro propio tiempo, que en $B C$ IV 45 estamos ante una versión literal; en el caso de lo que propone Apiano en $B C$ V 191 deberíamos decir entonces que no estamos ante una traducción (¿de qué texto?) sino ante la paráfrasis de unos contenidos conocidos por vía indirecta.

Pero quizá lo más propio sea intentar aclarar el asunto desde las propias observaciones antiguas sobre la traducción. Empezaremos por recordar lo que comenta Cicerón (De optimo genere oratorum 14) hablando sobre su propias traducciones de Esquines y Demóstenes ${ }^{22}$ :

Nec conuerti ut interpres, sed ut orator, sententiis isdem et earum formis tamquam figuris, uerbis ad nostram consuetudinem aptis. In quibus non uerbum pro uerbo necesse habui reddere, sed genus omne uerborum uimque seruaui. Non enim ea me adnumerare lectori putaui oportere sed tamquam appendere.

En la restante obra de Cicerón (cf. p. e. Fin. I 6, III 15), así como en algún lugar de Horacio (Ep. II 3.133: nec uerbo uerbum curabis reddere fidus interpres), se encuentran declaraciones similares, al menos por lo que a la valoración del papel del interpres se refiere. Con todo, el segundo pasaje fundamental para conocer las ideas antiguas sobre la traducción se halla en la Epístola LVII (5.2) de San Jerónimo:

Ego enim non solum fateor, sed libera uoce profiteor, me in interpretatione Graecorum, absque scripturis sanctis, ubi et uerborum ordo mysterium est, non uerbum e uerbo, sed sensum exprimere de sensu.

Tanto Cicerón como San Jerónimo plantean una dicotomía, en el caso del primero entre el interpres y el orator. El interpres, dice Cicerón, realiza una traducción exacta del tipo uerbum pro uerbo. El orator, en cambio, no atiende tanto a la literalidad de la versión como a mantener genus omne uerborum uimque. Brock considera que por interpres hemos de entender, concretamente, el traductor profesional que producía «slavish renderings of legal and business documents» ${ }^{23}$; en cambio, el orator es, en el pasaje de De opti-

22 Tomo las evidencias sobre Cicerón, Horacio y San Jerónimo (cf. infra) de S. Brock, «Aspects of Translation Technique in Antiquity», GRBS 20, 1979, pp. 69-87 (cf. p. 69).

${ }_{23}$ Cf. Brock, «Aspects of Translation ...», p. 69. En el mismo sentido, cf. lo que comen-

EMERITA (EM) LXXIV 1, enero-junio 2006 pp. 17-28 ISSN 0013-6662 
mo genere oratorum, evidentemente Cicerón; más en general, según la interpretación de Brock, el término orator debe abarcar a todo aquel que practica la traducción de un texto literario o, mejor dicho, la traducción literaria. A San Jerónimo le debemos las expresiones que tipifican los dos estilos de versión, pues, como él dice, es posible traducir uerbum e uerbo (cf. uerbum pro uerbo en el texto de Cicerón) o bien sensum ... de sensu. Podemos recordar la caracterización que hace Brock de uno y otro estilo: «The sensus de sensu approach can be seen as bringing the original to the reader, whereas in the uerbum e uerbo translation (...) the psychological effect is to bring the reader to the original» ${ }^{24}$.

Yo entiendo, como ya he dicho antes, que tanto en BC IV 45 como en V 191 Apiano concibe su actividad como "traducir", $\mu \varepsilon \tau \alpha \beta \alpha \lambda \varepsilon i v$. Pero creo también que en el primer caso, que precisamente es traducción de un texto legal, pretende ser un fidus interpres ${ }^{25}$ que aspira a traducir uerbum e uerbo: "El escrito de proscripción de los triunviros era de este tenor, en la medida en que se puede verter a la lengua griega desde la latina”. En cambio, en V 191, Apiano es claramente un orator en el sentido propuesto por Brock, que reconstruye con inevitable libertad (cf. tipo sensus de sensu), a partir de las informaciones recogidas en los $\dot{\tau} \pi \circ \nu_{\eta} \mu_{\alpha} \tau \alpha$, los dos discursos que quiere transmitir a sus lectores: "Esto se dijeron el uno al otro, según se podía verterlo a partir de los comentarios, de acuerdo con las posibilidades de esta lengua, deduciéndolo yo de la idea general de lo dicho" ${ }^{26}$.

7. Llegados a este punto, y de cara a seguir apreciando el proceder de Apiano como traductor, cabría continuar por dos caminos distintos. Una vía posible de estudio consiste en centrarse en $B C$ V 191 y analizar las cualidades de Apiano como orator, en el sentido que parece adquirir este sustantivo en el texto de Cicerón antes citado. Ahora bien, éste es un camino que considero preferible obviar por dos motivos. De un lado, parece a priori complejo analizar la elaboración a que sometió Apiano los parlamentos incluidos entre $B C$ V 176 y 190 cuando carecemos de una versión alternativa de los dis-

\footnotetext{
ta sobre el interpres en Cicerón L.C. Pérez Castro, «Acerca de las terminologías ciceronianas: préstamos, calcos y correspondencias», EMERITA 70, 2002, pp. 205-212 (cf. p. 208).

24 Cf. Brock, «Aspects of Translation ...», p. 73.

25 Cf. Hor., Ep. II 3, 133.

26 La cursiva es mía en el caso de las dos traducciones (BC IV 45 y V 191).
} 
cursos con la que cotejar el texto del alejandrino. Por otro lado, el tema de los discursos en la obra del historiador ya ha sido tratado en repetidas ocasiones, de tal modo que parece poco probable que se pudieran alcanzar conclusiones novedosas prosiguiendo por este camino ${ }^{27}$.

Aún queda abierta la posibilidad de estudiar a Apiano en cuanto interpres, es decir, en cuanto traductor según nuestro sentido actual de la palabra, tal y como lo vemos operar entre $B C$ IV 31 y 45 . Pero ha de reconocerse de entrada que, al querer desarrollar esta vía de estudio, nos encontramos otra vez con el problema que ya conocemos: el texto de la proscripción no nos es conocido en su original latino, y tampoco podemos confrontar la traducción de Apiano con otros textos griegos que transmitan versiones alternativas de esta $\pi \rho \gamma_{\rho} \alpha \varphi \eta^{28}$.

8. Con todo, pienso que es posible efectuar algunas observaciones sobre la manera en que Apiano traduce del latín a partir del análisis de su texto griego. Por ejemplo, es evidente que, en la redacción de $B C$ IV 31 - 45, Apiano se ha esforzado por encontrar equivalentes griegos para términos que debían de aparecer en el texto latino expresando instituciones o conceptos culturales romanos. Podemos revisar tres casos procedentes de la narración sobre el asesinato de César (BC IV 34) ${ }^{29}$ :

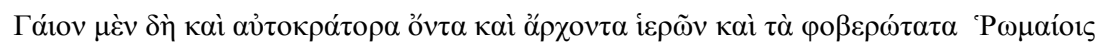

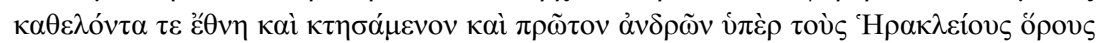

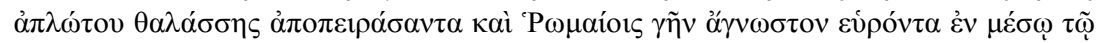

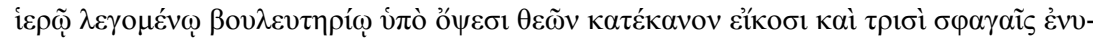

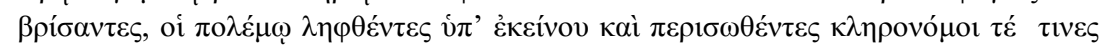

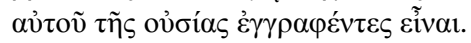

a) En el pasaje citado se dice que a César lo mataron $\dot{\varepsilon} \nu \mu \varepsilon \dot{\varepsilon} \sigma \omega$ $\tau \tilde{\varphi}$

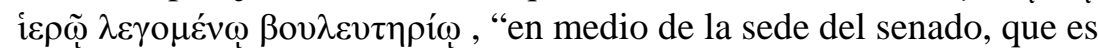

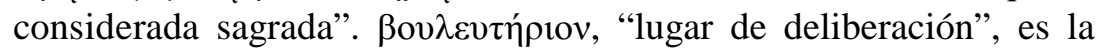
voz escogida por Apiano para referirse al edificio donde se reunía el senado. Con el sentido derivado de 'consejo' o 'senado' (grupo de personas que integran el senado) la palabra aparece también en otros

27 Cf. lo que apunto, a manera de ejemplo, en n. 11.

28 Cf. lo que se indica en n. 10.

29 El orden en que discuto los tres casos no coincide con el orden en que aparecen éstos en el texto de Apiano. Cito el texto griego por la edición de Mendelssohn y Viereck (cf. n. 9).

EMERITA (EM) LXXIV 1, enero-junio 2006 pp. 17-28 ISSN 0013-6662 
textos y autores griegos (cfr. D.H. II 12), en competencia con $\gamma \varepsilon \rho o v-$ бía, el colegio de senes, que es la voz que escogen, por ejemplo, Plu-

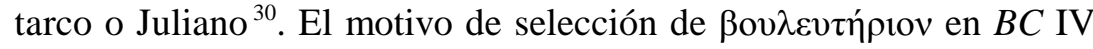
34, en detrimento de $\gamma \varepsilon \rho o v \sigma i ́ \alpha$, debió de ser el deseo de introducir una referencia local específica que aclarase la ambivalencia del término latino senatus, probablemente la palabra que aparecía en el texto original de la proscripción.

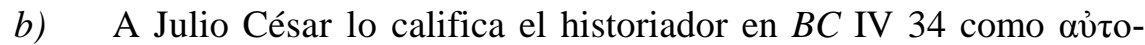

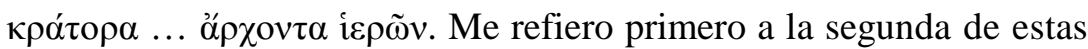
denominaciones. ó $\rho \omega v$ i $\varepsilon \rho \tilde{\omega} v$ parece ser una versión griega del título de Pontifex maximus que César poseyó del 63 al 44 a. C. Es cierto que otros autores traducen este cargo al griego por medio de otros giros ${ }^{31}$; no obstante, resulta difícil imaginar qué otro término latino podría esconderse tras ó $\rho \chi \omega v$ i $\varepsilon \rho \tilde{v} v$. Da más bien la impresión de que la distinta organización religiosa de Grecia, que no conocía una autoridad religiosa suprema, llevó a los griegos a inventar nombres distintos para la magistratura romana. Por este motivo, a falta de un término propio, Apiano acuña en este pasaje ${ }^{32}$ la expresión de carácter general ó $\rho \chi \omega v$ i $\varepsilon \rho \tilde{\omega} v$, "el que manda en los asuntos sagrados", expresión que, aun debiendo de ser fiel al texto de partida, carecía evidentemente del contenido específico de Pontifex maximus.

c) Merece atención también el hecho de que el pasaje denomine a Julio César como av่ esta palabra es 'dueño de sí mismo' ${ }^{34}$; de aquí procede (cf. ibíd.) el significado derivado de 'quien tiene autoridad absoluta', significado

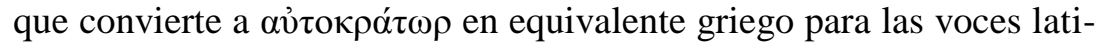

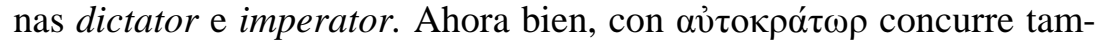

30 Cf. Plu. II 789 e; Jul., Or. II 97 b. En Apiano, la voz $\gamma \varepsilon \rho o v \sigma i ́ \alpha$ se refiere habitualmente al senado de los cartagineses.

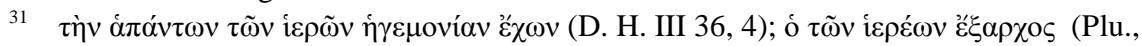
Num. 10). Cf. Famerie, Le latin et le grec..., pp. 90-91.

32 En la obra de Apiano, la expresión óp $\chi \omega v$ i $\varepsilon \rho \tilde{\omega} v$ sólo aparece en este lugar (cfr. Famerie, Concordantia...., s.v.)

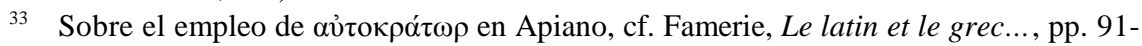
102.

34 Cf. F.R. Adrados y E. Gangutia (eds.), Diccionario griego español (DGE), Madrid, CSIC, 1980-, s.v. 
bién en griego $\delta \iota \kappa \tau \alpha ́ \tau \omega \rho$, latinismo atestiguado ya en la obra de Polibio (III 87, 6). Parece curioso que, si el decreto de proscripción denominaba a César dictator, Apiano haya preferido traducir el título como

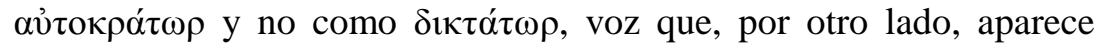
repetidamente en la Historia Romana ${ }^{35}$. Por ello cabe pensar que el texto latino de la proscripción no se refería a César como dictator sino que le aplicaba la denominación de imperator. La interpretación de

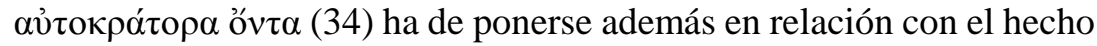
de que, en un lugar posterior de esta traducción (39), el texto hable de

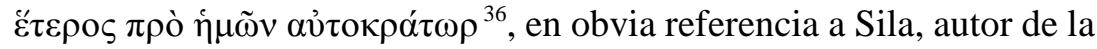
primera proscripción en el año 82 a. C.; quizá no resultaba fuera de lugar que los promotores de la proscripción del año 43 a. C. llamaran a su precursor alter ante nobis imperator ${ }^{37}$.

Creo que estos ejemplos pueden bastar para mostrar la preocupación de Apiano por ofrecer en $B C$ IV 31 - 45 una traducción próxima al tipo uerbum

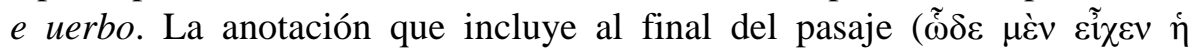

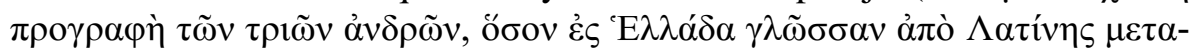
$\beta \alpha \lambda \varepsilon \tilde{v}$ ) es notable además por la conciencia de traductor que implica, una conciencia de traductor a la que tampoco parece ajeno el orgullo por la labor realizada.

9. Si ésta es la única traducción uerbum e uerbo elaborada por Apiano e introducida en la obra, puede entenderse perfectamente que incluyera en $B C$ IV 45 la declaración que acabamos de discutir. Pero, ¿por qué habla Apiano en un tono parecido en $B C \mathrm{~V} 191$ ? Que este caso lo consideraba también como traducción ya ha sido aclarado. El problema es otro y puede plantearse así: la adaptación de las fuentes efectuada entre $B C$ V 176 y 190, ¿poseía alguna característica singular que la diferenciaba de otras adaptaciones de discursos incluidas en la Historia romana?; ¿por qué incluye Apiano sólo en este caso

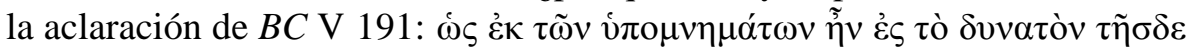

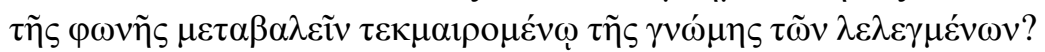

35 Sobre $\delta ı \kappa \tau \alpha ́ \tau \omega \rho$ en Apiano, cf. Famerie, Le latin et le grec ..., pp. 110-122.

36 Dentro de la traducción de la $\pi \rho \gamma \gamma \rho \alpha \varphi \eta ́$, éstas son las dos únicas ocasiones en que

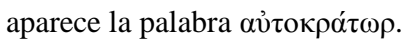

37 Recuerdo que la denominación de imperator se le aplica a Sila en monedas de su época. Cf. Fröhlich, "L. Cornelius [392] Sulla”, RE IV.1 (1900), cols. 1522-1566 (cf. col. 1557); M.H. Crawford, Roman Republican Coinage. I, Cambridge, 1974, pp. 373-374. 
En relación con esta cuestión no podemos ir más allá de las meras hipótesis. Yo propongo que el historiador quiere resaltar con esta expresión su fidelidad a unos comentarios ( ponía con un detalle mayor de lo habitual las líneas maestras de los discursos pronunciados por Lucio Antonio y Octavio. La hipótesis cobra fuerza si es verdad, como sugiere entre otros Gowing (cf. n. 13), que la fuente de este pasaje de Apiano son las Memorias del propio Octavio Augusto ${ }^{38}$, uno de los protagonistas de la escena.

10. Las traducciones antiguas del latín al griego de las que tenemos noticia a día de hoy no son demasiado abundantes ${ }^{39}$. Algunas se han perdido, como por ejemplo aquéllas a las que me refería en la nota 5. Las que conservamos proceden, además, de una cronología relativamente tardía, del siglo cuarto en adelante ${ }^{40}$. Por estos motivos parece que no se ha de desdeñar el testimonio de Apiano en cuanto traductor del latín al griego en el siglo segundo de nuestra era. Si en casos como los de Polibio, Pisandro o Arriano (cf. n. 5) hemos perdido la traducción griega, lo peculiar en el caso de Apiano es que lo que se ha perdido no es la versión sino el original latino.

Con arreglo a las normas editoriales vigentes para las publicaciones periódicas del CSIC, se hace constar que el original definitivo de este artículo se recibió en la redacción de EMERITA en el segundo semestre de 2005, siendo aprobada su publicación en ese mismo período (29.9.05 - 11.12.05).

38 Citadas como fuente en distintos lugares de las Vidas paralelas de Plutarco: cf. p. ej. Brut. 41; Ant. 22. Famerie (Le latin et le grec..., pp. 30-31) se muestra muy escéptico sobre la posibilidad de identificar la fuente del pasaje.

39 Un tipo especial, que merece estudio aparte, lo constituyen las traducciones oficiales, del tipo representado por la versión griega de las Res gestae diui Augusti (cf. Rochette, "Bilinguisme...", pp. 8-16); frente a estas versiones, la incluida en BC IV 31-45 no es una traducción oficial sino la traducción que un particular hace de un texto oficial. De cara a la historia de la traducción (y de la educación) poseen también importancia las traducciones escolares conservadas en papiros, sobre las cuales cf. Fisher, “Greek Translations...”, pp. 183-189; Rochette, "Bilinguisme...", pp. 16-19.

40 En general se trata de traducciones de autores cristianos, elaboradas por Eusebio de Cesarea, Sofronio, Peanio, más algún traductor anónimo (cf. Fisher, "Greek Translations...", pp. 189-207). De un contexto cristiano procede además la traducción de la Égloga cuarta virgiliana de la que hablábamos al principio de este trabajo.

EMERITA (EM) LXXIV 1, enero-junio 2006 pp. 17-28 ISSN 0013-6662 